\title{
Mathematical Modeling and Dynamic Analysis of Planetary Gears System with Time-Varying Parameters
}

\author{
Zhengming Xiao $\mathbb{D}$, Jinxin Cao, and Yinxin Yu \\ Faculty of Mechanical and Electrical Engineering, Kunming University of Science and Technology, Kunming, Yunnan, China \\ Correspondence should be addressed to Zhengming Xiao; suzem@sina.com
}

Received 14 January 2020; Accepted 20 February 2020; Published 16 March 2020

Guest Editor: Jian Huang

Copyright (c) 2020 Zhengming Xiao et al. This is an open access article distributed under the Creative Commons Attribution License, which permits unrestricted use, distribution, and reproduction in any medium, provided the original work is properly cited.

\begin{abstract}
Planetary gears are widely used in automobiles, helicopters, heavy machinery, etc., due to the high speed reductions in compact spaces; however, the gear fault and early damage induced by the vibration of planetary gears remains a key concern. The timevarying parameters have a vital influence on dynamic performance and reliability of the gearbox. An analytical model is proposed to investigate the effect of gear tooth crack on the gear mesh stiffness, and then the dynamical model of the planetary gears with time-varying parameters is established. The natural characteristics of the transmission system are calculated, and the dynamic responses of transmission components, as well as dynamic meshing force of each pair of gear are investigated based on varying internal excitations induced by time-varying parameters and tooth root crack. The effects of gear tooth root crack size on the planetary gear dynamics are simulated, and the mapping rules between damage degree and gear dynamics are revealed. In order to verify the theoretical model and simulation results, the planetary gear test rig was built by assembling faulty and healthy gear separately. The failure mechanism and dynamic characteristics of the planetary gears with tooth root crack are clarified by comparing the analytical results and experimental data.
\end{abstract}

\section{Introduction}

Planetary transmissions are an important form of mechanical transmission. Because of its advantages of high transmission ratio, high bearing capacity, and compact structure, it is widely used in complex mechanical equipment such as aerospace, wind power generation equipment, and mining machinery. Due to the influence of time-varying parameters, the planetary gear system always has the problem of nonlinear dynamics and excessive vibration. The planetary transmission system has a high failure rate, and the root crack is one of the most important forms of gear failure, due to complicated internal structure and large load during operation. When the root of the transmission system is cracked, the vibration of the system will be intensified and, in serious cases, the equipment will be damaged. Therefore, the dynamic study of the planetary gear transmission system with tooth root crack failure and analysis of the fault mechanism has an important theoretical significance and engineering application value for improving the reliability and service life of the gear. Mathematical modeling and analysis are important methods to solve the nonlinear dynamics of planetary gear transmission systems. The control of nonlinear dynamic systems is a widely recognized challenging issue. It is promising to develop vibration reduction design of the planetary gear system based on U-model, because of the unique advantages of $U$-model in nonlinear control $[1,2]$. Some scholars have carried out a lot of work on the dynamics of gear systems by mathematical modeling. Bonori and Pellicano [3] presented a dynamic model of a single pair of gear transmission systems and analyzed the effects of random manufacturing errors on the dynamic response of the system. Chaari et al. [4] established an analytical model of time-varying gear meshing stiffness based on the analytical method and analyzed the variation of crack to gear stiffness under two different parameters. Guo and 
Parker [5] established a dynamic model of the planetary gear train based on the lumped parameter method and solved the dynamic model to obtain the dynamic response of the transmission system. Chen and Shao [6] studied the effects of internal ring cracks on the timevarying mesh stiffness and dynamic response of planetary gear trains. Some scholars have also carried out a lot of research on the dynamics of planetary transmission systems. Zhou [7] studied the influence of crack parameters on dynamic characteristics by establishing a finite element model. Wan [8] proposed a meshing stiffness correction method based on the potential energy method. The dynamic model of a gear transmission system with tooth root crack was established by using the lumped parameter method, and the dynamic equation was solved to obtain the dynamic response. In previous planetary transmission dynamics analysis, the bearing was simplified to the ideal constraint boundary, and the bearing was simplified to a constant stiffness coefficient spring, also ignoring the influence of the bearing timevarying stiffness on the dynamic characteristics of the transmission system. Bearings and gears are the key components of the planetary transmission system. The dynamic characteristics of the bearings have an important influence on the stability and service life of the transmission system. Walters [9] proposed the dynamic analysis model of the rolling bearing. The dynamic model of the rolling bearing was established, and the drag force between the rolling element and the ferrule was obtained by considering the four degrees of freedom of the rolling element and the six degrees of freedom of the cage. Harris and Kotzalas [10] considered the effect of elastohydrodynamics on the basis of quasi-static analysis and improved the pseudostatic analysis method of rolling bearings. In establishing the balance equations of the rolling element, the cage, and the inner ring, the rolling element's revolution and bearing deformation parameters are obtained.

Zhou [11] considered the time-varying stiffness of rolling bearings, established the coupled dynamics model of MWclass wind turbine gear transmission system by using the lumped parameter method, obtained the inherent characteristics of the transmission system, and solved the dynamics of each bearing contact stress, but it does not consider the influence of the centrifugal force of the roller on the bearing stiffness. Mohammed et al. [12] presents an investigation of the performance of crack propagation scenarios, to compare these scenarios from a fault diagnostics point of view. Park et al. [13] proposes a variance of energy residual (VER) method, for planetary gear fault detection under variable-speed conditions.

In the planetary gear transmission system, the dynamic meshing excitation of the gear teeth is transmitted to the casing through the bearing, causing vibration and noise of the casing. The dynamic characteristics of the bearing have an important influence on the performance of the entire transmission system. In the past, the scholars neglected the influence of the mass of the rolling element and the centrifugal force of the roller on the time-varying stiffness of the

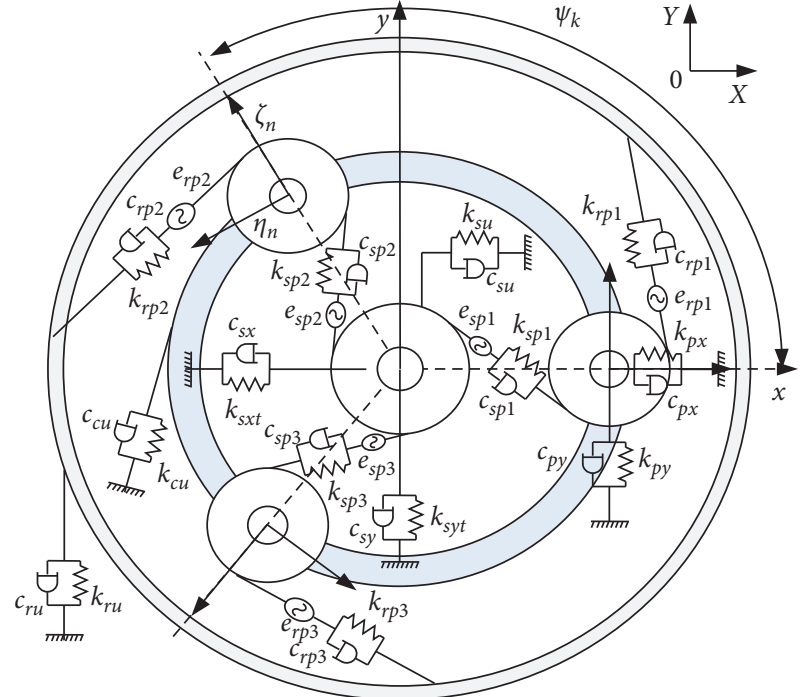

FIgURE 1: Mechanical model of planetary gear system.

bearing when studying the gear-bearing coupling dynamic system. At the same time, the dynamic characteristics of the faulty planetary transmission system were not studied. In this paper, the planetary transmission system with tooth root crack is taken as the research object. According to the Hertz contact theory, considering the mass of the rolling element and the effect of centrifugal force, the time-varying bearing stiffness model is established. At the same time, the timevarying bearing stiffness, the transmission error, and the time-varying meshing stiffness of the cracked gear are introduced. The gear-bearing coupling dynamics model with crack is established. The influence of time-varying bearing stiffness on the dynamic characteristics of the gear transmission system is analyzed. The influence of crack failure on the dynamic characteristics of the gear is explored, which provides a more detailed mathematical method for the fault diagnosis and vibration control of the planetary gear transmission system.

\section{Dynamic Model of Planetary Gear System}

The dynamic characteristics of the planetary gear transmission system are affected by many factors. The mechanical model is simplified, as shown in Figure 1. It is assumed that the three planet gears in the planetary transmission system are evenly distributed along the circumference, and the geometric parameters and physical parameters of the three planet gears are the same. The engagement between the gear pairs is simplified to a spring with time-varying stiffness. Considering the gear meshing error, the time-varying bearing stiffness of the bearing, and the time-varying meshing factor of the gear, the planetary gear translation-torsion dynamics model is established by the lumped parameter method. Each component has 2 translational degrees of freedom and 1 torsional degree of freedom, and the model has 18 degrees of freedom. 
Ring: $\left\{\begin{array}{l}m_{r}\left(\ddot{x}_{r}-2 \omega_{c} \dot{y}_{r}-\omega_{c}^{2} x_{r}\right)-\sum_{n=1}^{N}\left(c_{r n} \dot{\delta}_{r n}+k_{r n} \delta_{r n}\right) \sin \psi_{r n}+c_{r x} \dot{x}_{r}+k_{r x} x_{r}=0, \\ m_{r}\left(\ddot{y}_{r}+2 \omega_{c} \dot{x}_{r}-\omega_{c}^{2} y_{r}\right)+\sum_{n=1}^{N}\left(c_{r n} \dot{\delta}_{r n}+k_{r n} \delta_{r n}\right) \cos \psi_{r n}+c_{r y} \dot{y}_{r}+k_{r y} y_{r}=0, \\ \left(\frac{I_{r}}{r_{r}^{2}}\right) \ddot{u}_{r}+\sum_{n=1}^{N}\left(c_{r n} \dot{\delta}_{r n}+k_{r n} \delta_{r n}\right)+c_{r u} \dot{u}_{c}+k_{r u} u_{c}=\frac{T_{r}}{r_{r}},\end{array}\right.$

Sun: $\left\{\begin{array}{l}m_{s}\left(\ddot{x}_{s}-2 \omega_{c} \dot{y}_{s}-\omega_{c}^{2} x_{s}\right)-\sum_{n=1}^{N}\left(c_{s n} \dot{\delta}_{s n}+k_{s n} \delta_{s n}\right) \sin \psi_{s n}+c_{s x} \dot{x}_{s}+k_{s x t} x_{s}=0 \\ m_{s}\left(\ddot{y}_{s}+2 \omega_{c} \dot{x}_{s}-\omega_{c}^{2} y_{s}\right)+\sum_{n=1}^{N}\left(c_{s n} \dot{\delta}_{s n}+k_{s n} \delta_{s n}\right) \cos \psi_{s n}+c_{s y} \dot{y}_{s}+k_{s y t} y_{s}=0 \\ \left(\frac{I_{s}}{r_{s}^{2}}\right) \ddot{u}_{s}+\sum_{n=1}^{N}\left(k_{s n} \delta_{s n}+c_{s n} \dot{\delta}_{s n}\right)+c_{s u} \dot{u}_{s}+k_{s u} u_{s}=\frac{T_{s}}{r_{s}},\end{array}\right.$

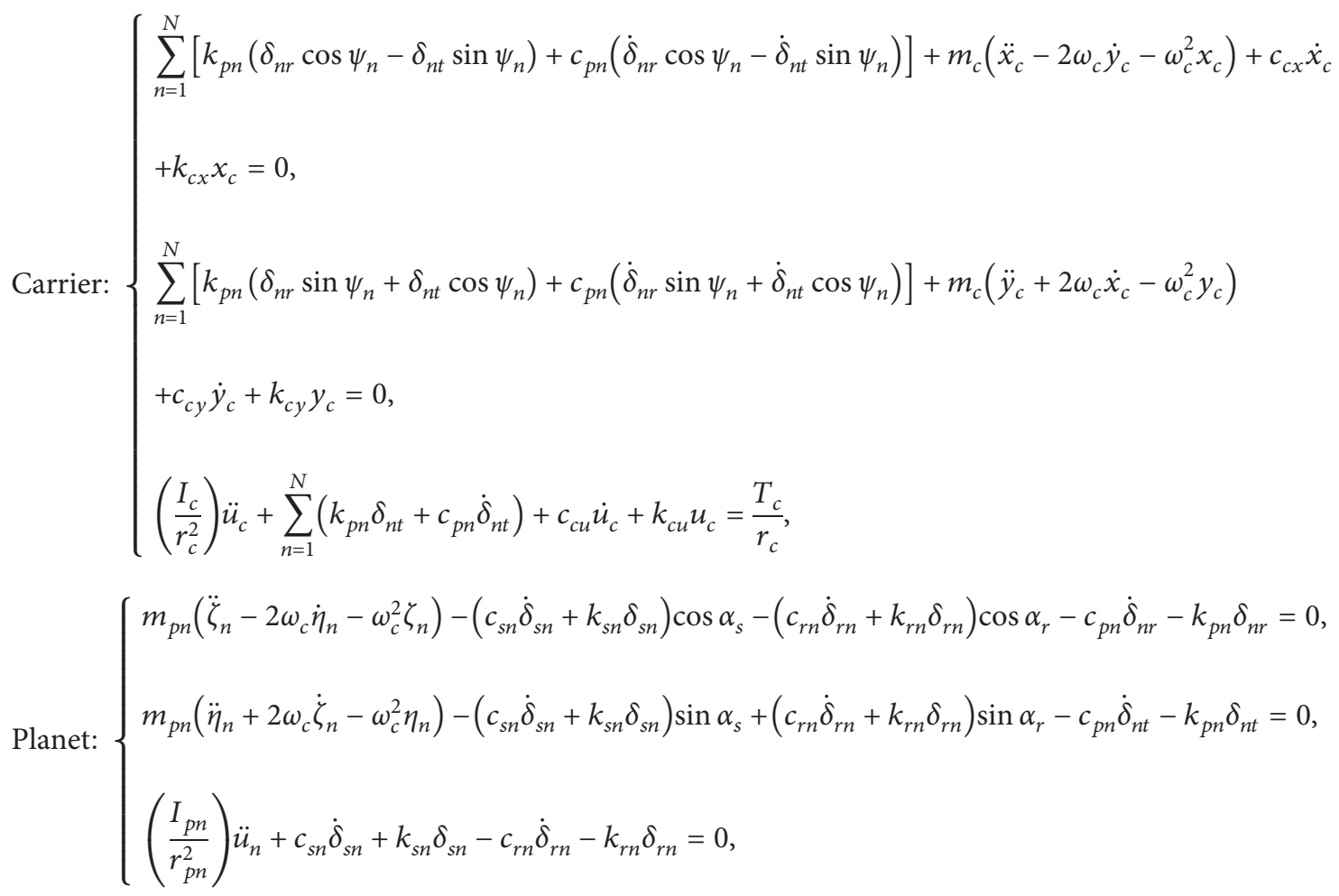

where $\delta_{s n}$ is the elastic deformation of the sun gear and the $n$th planet gear on the meshing line:

$$
\begin{aligned}
\delta_{s n}= & -x_{s} \sin \psi_{s n}+y_{s} \cos \psi_{s n}-\zeta_{n} \sin \alpha_{s}-\eta_{n} \cos \alpha_{s} \\
& +u_{s}+u_{n}+e_{s n},
\end{aligned}
$$

where $\delta_{r n}$ is the elastic deformation of the ring and the $n$th planet gear on the meshing line:

$$
\begin{aligned}
\delta_{r n}= & y_{r} \cos \psi_{r n}-x_{r} \sin \psi_{r n}-\zeta_{n} \sin \alpha_{r}-\eta_{n} \cos \alpha_{r} \\
& +u_{r}-u_{n}+e_{r n},
\end{aligned}
$$

where $\delta_{n r}$ is the projection of the planet carrier in the $\zeta$ direction relative to the $n$th planet gear:

$$
\delta_{n r}=x_{c} \cos \psi_{n}+y_{c} \sin \psi_{n}-\zeta_{n}
$$


where $\delta_{n t}$ is the projection of the planet carrier in the $\eta$ direction with respect to the $n$th planet gear:

$$
\delta_{n t}=-x_{c} \sin \psi_{n}+y_{c} \cos \psi_{n}+u_{c}-\eta_{n},
$$

where $\psi_{s n}=\psi_{n}-\alpha_{s}, \psi_{r n}=\psi_{n}+\alpha_{r}, \psi_{n}$ is the position angle of the $n$th planet gear, $\alpha_{r}$ is the meshing angle between the ring and the planet gear, and $\alpha_{s}$ is the meshing angle between the sun gear and the planet gear.

The dynamic equations of the various components established above are collated. The dynamic equations of the planetary transmission system can be expressed in the form of a matrix. The dynamic equation of the transmission system can be expressed as follows:

$$
M \ddot{x}+\omega_{c} G \dot{x}+\left[K_{b}+K_{m}-\omega_{c}^{2} K_{\omega}\right] x=F+T,
$$

where $G$ is the gyromatrix; $K_{m}$ is the meshing stiffness matrix; $K_{\omega}$ is the centripetal stiffness matrix; $x$ is the generalized displacement matrix of the system; $M$ is the generalized mass matrix of the system; $K_{b}$ is the bearing support stiffness matrix; $K_{m}$ is the meshing stiffness matrix; $T$ is the external load of the system; and $F$ is the internal excitation.

\section{Time-Varying Stiffness Model of Bearing}

In order to simplify the calculation, it is assumed that the steel ball rotates at a constant speed and the movement between the inner and outer rings of the bearing is pure rolling. Regardless of the oil film stiffness under bearing lubrication, the bearing stiffness of the bearing can be analyzed according to Hertz contact theory.

As shown in Figure 2, during the movement of the bearing, the inner and outer rings of the bearing are subjected to the weight of the roller and the centrifugal force. Therefore, the forces of the contact between the roller and the inner and outer rings of the bearing are different. The contact force $Q_{o}$ between the roller and the outer ring is as follows:

$$
\begin{aligned}
& Q_{o}=Q-m g \sin \varphi_{k}+m R_{p} \omega_{c}^{2}, \\
& \omega_{c}=\frac{\pi\left(1-\left(D_{b} / D_{p}\right) \cos \beta\right) n_{s}}{60},
\end{aligned}
$$

where $\omega_{c}$ is the angular velocity of the cage, $Q$ is the load on the inner ring of the bearing, $m$ is the mass of the steel ball. The radius of the circle where the geometric center of the roller is located is $R_{p}, D_{b}$ is the diameter of the roller, $n_{s}$ is the rotational speed of the shaft, and $\beta$ is the contact angle.

According to Hertz contact theory, the relationship between the contact force $Q$ of the rolling bearing steel ball and the elastic approaching amount $\delta$ can be expressed as follows:

$$
Q=K_{c} \delta^{3 / 2}
$$

where $Q$ is the contact load of the steel ball and $K_{c}$ is the Hertz contact stiffness.

According to the force balance condition, the component forces $f_{x}$ and $f_{y}$ of the bearing $x$ and $y$ directions are equal to the sum of the component forces of the load $Q_{k}$ of

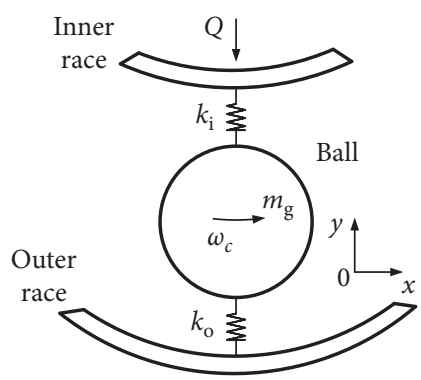

FIGURE 2: Bearing rolling element-ring model.

each roller in the $x$ and $y$ directions, and $f_{x}$ and $f_{y}$ can be expressed by the following formulas:

$$
\left\{\begin{array}{l}
f_{x}=\sum_{k=1}^{N} Q_{k} \cos \left(\varphi_{k}\right), \\
f_{y}=\sum_{k=1}^{N} Q_{k} \sin \left(\varphi_{k}\right) .
\end{array}\right.
$$

The radial stiffness $k_{b}$ of the bearing is

$$
k_{b}=\sum_{k=1}^{N}\left[K_{k} \cos \varphi_{k}\right] \text {, }
$$

where $K_{k}$ is the contact stiffness of the $k$ th roller.

The relevant parameters of the rolling bearing in the planetary transmission system are shown in Table 1. The bearing material is bearing steel, which is solved according to the time-varying bearing stiffness model of the rolling bearing. Figure 3 shows the variation of the radial stiffness of the bearing with the angle of the steel ball as the position angle changes.

\section{Effect of Crack on Gear Meshing Stiffness}

During the movement of the gear, the root position will produce a bending stress greater than other positions due to the periodicity of the load, which will easily lead to the occurrence of fatigue cracks in the gear teeth. When calculating the gear meshing stiffness, the gear teeth are generally considered be cantilever beams. There are five kinds of stiffness in the gear meshing pair: Hertz contact stiffness $k_{h}$, bending stiffness $k_{b}$, shear stiffness $k_{a}$, and radial compression stiffness $k_{s}$ [12]. In addition to the above stiffness, there is also a matrix flexible stiffness $k_{f}$ of the gear. When the gear is cracked, the surface contact area and radial force of the gear are constant, so the Hertz contact stiffness, radial compression stiffness, and matrix flexibility of the gear are unchanged. The gear meshing stiffness of the crack mainly considers the bending stiffness and shears stiffness.

In this paper, the planetary gearbox with tooth root crack fault is taken as the research object. The relevant parameters of the planetary box are shown in Table 2. When the crack tooth enters the meshing state, the meshing stiffness of the cracked gear is calculated. Figure 4 shows the comparison of the stiffness of a simulated crack occurrence (the tooth crack 
TABLE 1: Geometric parameters of the bearing.

\begin{tabular}{lcccc}
\hline Outer diameter $D(\mathrm{~mm})$ & Inner diameter $d(\mathrm{~mm})$ & Bearing width $B(\mathrm{~mm})$ & Ball diameter $D_{b}(\mathrm{~mm})$ & Number of rolling elements $n_{b}$ \\
\hline 80 & 40 & 18 & 12 & 9
\end{tabular}

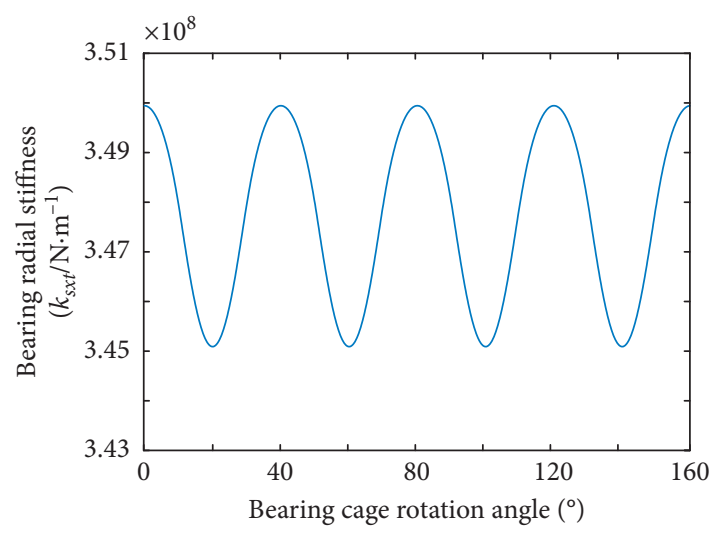

FIGURE 3: Time-varying bearing stiffness.

TABLE 2: Parameters of planetary gearbox.

\begin{tabular}{lcccc}
\hline Parameter & Ring & Carrier & $\begin{array}{c}\text { Planet } \\
\text { gear }\end{array}$ & Sun gear \\
\hline Mass $(\mathrm{kg})$ & 10.12 & 2.7 & 0.45 & 0.8 \\
Number of teeth & 71 & & 42.29 & 28 \\
Base circle diameter & 150.12 & 112 & 20 & 59.2 \\
$(\mathrm{~mm})$ & & & 2.25 & \\
Modulus (mm) & & & $20^{\circ}$ & \\
Engagement angle $\left(^{\circ}\right)$ & & &
\end{tabular}

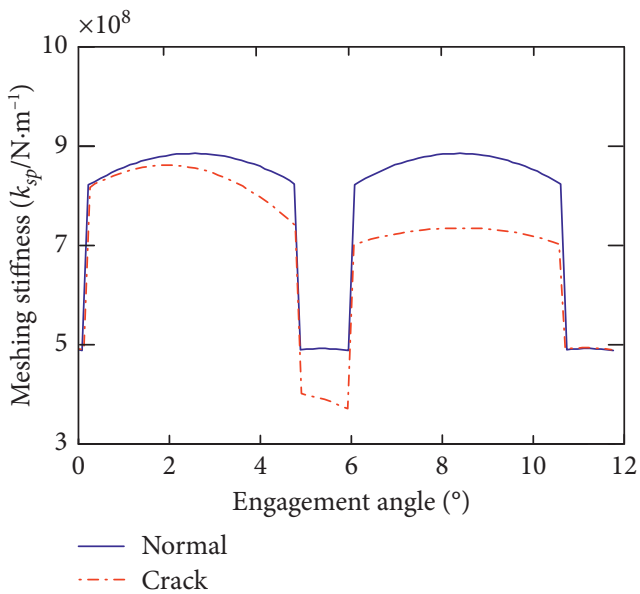

FIgURE 4: Time-varying meshing stiffness.

has an expansion angle $\alpha_{c}$ of $60^{\circ}$ and a crack depth $q$ of $1.8 \mathrm{~mm}$.) when engaged with a healthy gear pair.

\section{Dynamic Response and Analysis}

5.1. Modal Analysis of Planetary Gears. The modal analysis of the gear-bearing coupling dynamics model of the above mentioned planetary transmission system is carried out.
Regardless of the effect of damping, the average stiffness is used instead of the time-varying stiffness, and the dynamic equation is expressed as follows:

$$
M \ddot{x}+\omega_{c} G \dot{x}+\left[K_{b}+K_{m}-\omega_{c}^{2} K_{\omega}\right] x=0 .
$$

Thus, the $n$ positive real roots $\omega_{i}$, which are the natural frequencies of the system, could be determined. The main mode equation is

$$
\omega_{i}^{2} M \phi_{i}=\left[K_{b}+K_{m}\right] \phi_{i}
$$

The planetary gears system has 18 degrees of freedom. The natural frequencies of the system are calculated, as shown in Table 3, and the corresponding vibration modes are shown in Figure 5. There are three kinds of vibration mode: rigid mode, rotational modes (no repetitive frequency, no translation only torsion), and translational modes (there is a repetitive frequency, no torsion only translation). The phenomenon of 5 equivalent natural frequencies (repetitive frequency) occurs in the system because the geometrical and physical parameters of the planetary gears in the transmission system are assumed to be the same, resulting in a symmetrical type of the planetary transmission stage. The occurrence of repetitive frequencies causes different types of vibration to be induced at one frequency.

5.2. Dynamic Response Analysis of Planetary System. In order to deeply study the influence of sun gear root crack failure on the dynamic characteristics of the planetary gear transmission system. This paper selects the fault model and faultfree model of the sun gear crack $1.8 \mathrm{~mm}$. Considering the time-varying meshing stiffness of healthy and tooth cracked gears, the directional parameter method is used to establish the translation-torsion dynamics modeling of the planetary transmission system. The dynamic equation is solved by Runge-Kutta method. The input torque of the system is $30 \mathrm{Nm}$, and the dynamic response of the planetary transmission system at $1000 \mathrm{r} / \mathrm{min}$ is analyzed. The meshing frequency of the transmission system is $334.68 \mathrm{~Hz}$, and the bearing ball revolution frequency is $118.1 \mathrm{~Hz}$.

Figures 6-8 shows the vibration velocity response of a healthy and root-cracked gear transmission component. As shown in Figure 6, the peak velocity of the sun gear of the healthy system is $5.25 \mathrm{~mm} / \mathrm{s}$, and the peak velocity of the sun gear with root crack fault transmission system is $8.77 \mathrm{~mm} / \mathrm{s}$. As shown in Figure 7, the peak velocity of the planet gear of the healthy system is $2.83 \mathrm{~mm} / \mathrm{s}$, and the peak velocity of the sun gear with root crack fault transmission system is $3.95 \mathrm{~mm} / \mathrm{s}$. As shown in Figure 8, the peak velocity of the ring gear of the healthy system is $0.295 \mathrm{~mm} / \mathrm{s}$, and the peak velocity of the ring gear with the root crack fault transmission system is $0.362 \mathrm{~mm} / \mathrm{s}$.

Through the comparison of the time domain waveform of the healthy and faulty transmission systems, it can be seen 
TABLE 3: Natural frequencies of the system.

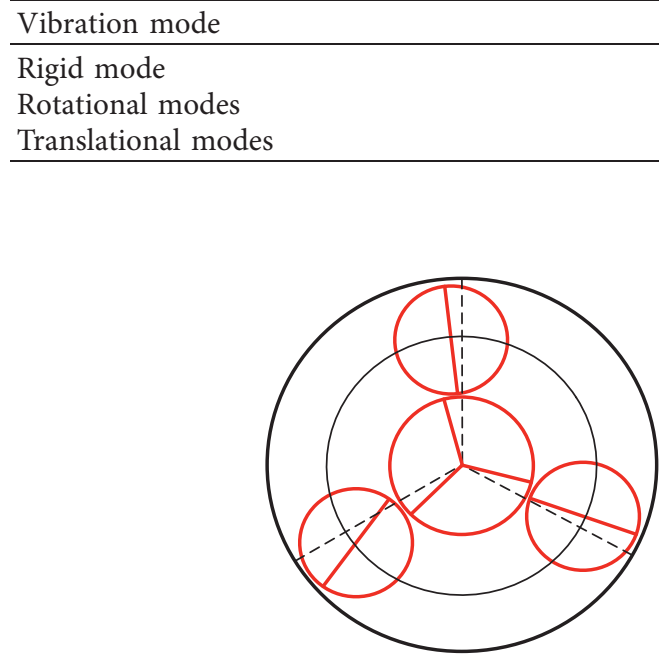

(a)

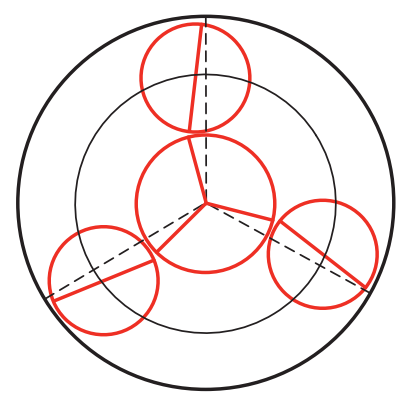

(b)

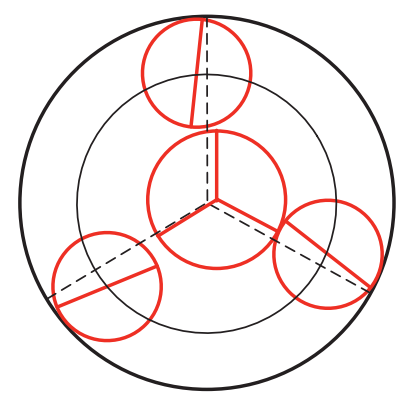

(c)

FIgURE 5: Vibration modes of planetary transmission system: (a) rigid mode, (b) rotational mode, and (c) translational mode.

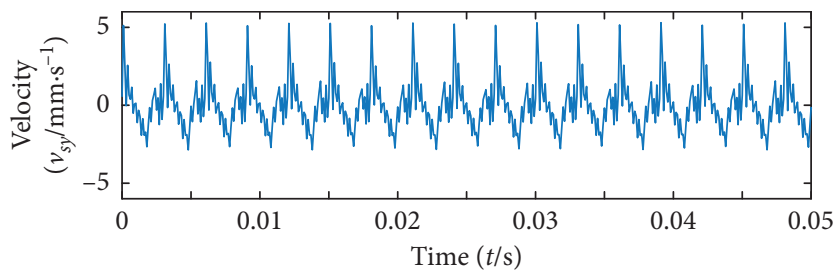

(a)

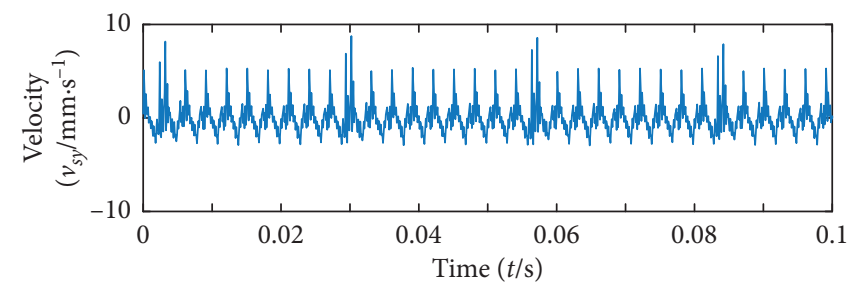

(b)

Figure 6: Vibration velocity response of the sun gear. (a) Healthy planetary transmission system. (b) Faulty planetary transmission system.

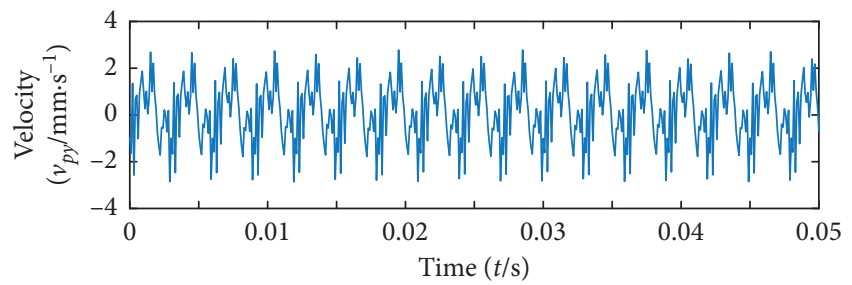

(a)

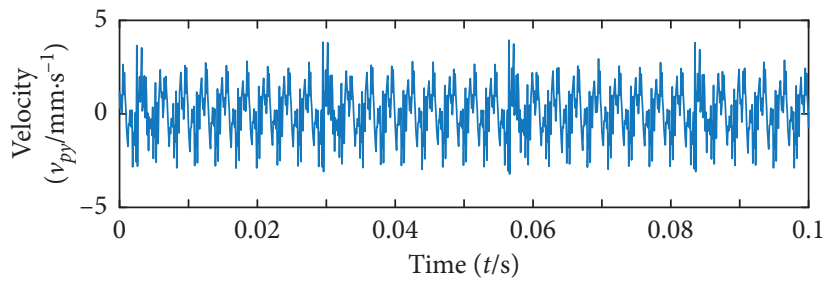

(b)

FIGURE 7: Vibration velocity response of planet gear. (a) Healthy planetary transmission system. (b) Faulty planetary transmission system.

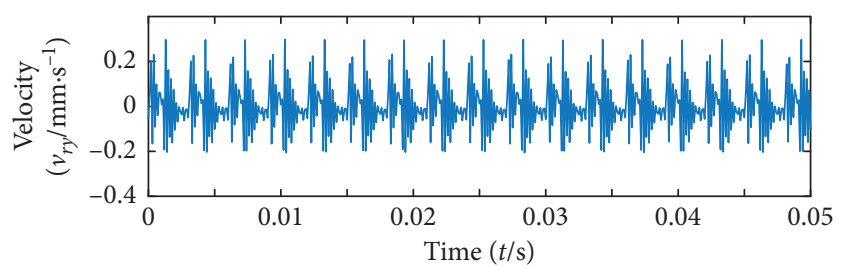

(a)

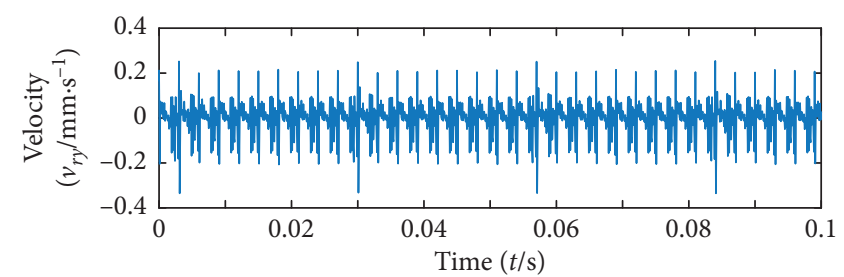

(b)

FIGURE 8: Vibration velocity of ring. (a) Healthy planetary transmission system. (b) Faulty planetary transmission system. 


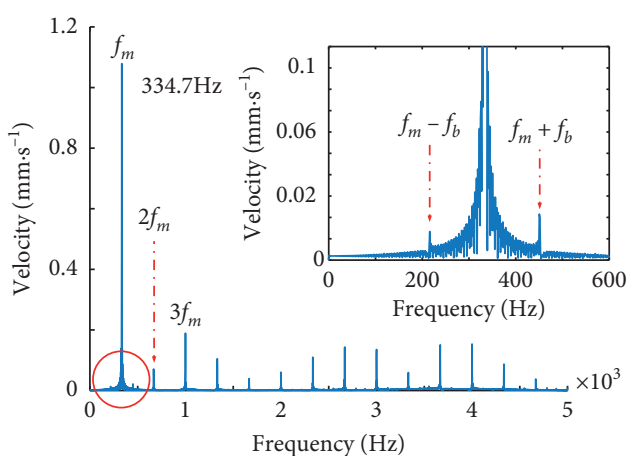

(a)

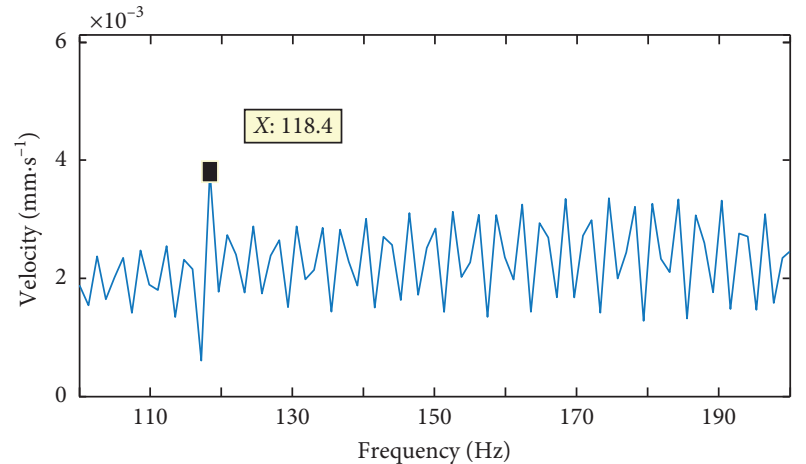

(b)

FIGURE 9: Spectrum diagram of sun gear. (a) Spectrum of healthy signal. (b) Spectrum refinement of healthy signals.

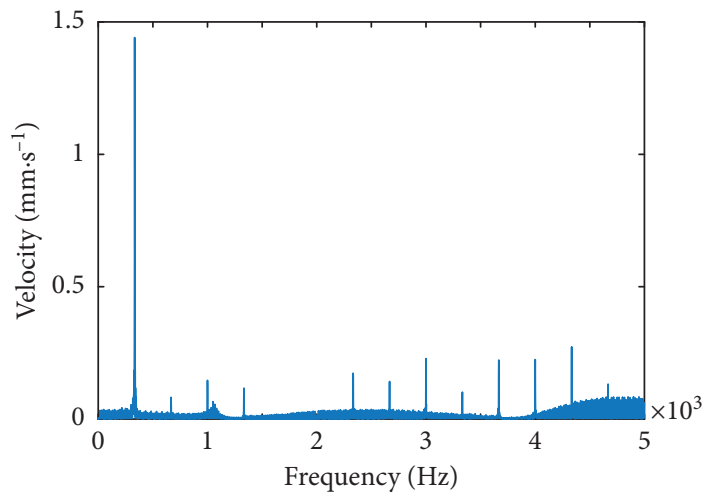

(a)

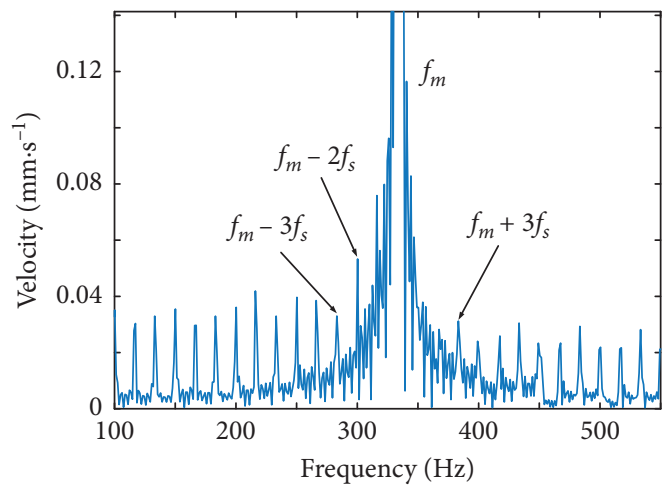

(b)

Figure 10: Spectrum diagram of sun gear with root crack. (a) Spectrum of fault signal. (b) Spectrum refinement of fault signal.

that a periodic shock signal appears in the time domain waveform diagram of the faulty system compared to the healthy transmission system. The occurrence of sun gear cracks causes the gear teeth of the cracks to reduce the meshing stiffness, which makes the vibration velocity the sun gear, the planet gears, and the ring in the system increase significantly, which aggravates the vibration of the planetary transmission system.

The spectrum analysis of the vibration velocity response of the sun gear in the $y$ direction of the transmission system is performed. Figures 9 and 10 are the spectrum diagrams of the vibration velocity response of the healthy and the tooth root crack faulty transmission system. As shown in Figure 9, in the spectrum diagram of the healthy transmission system, the meshing frequency of the transmission system is $334.7 \mathrm{~Hz}$, and the bearing ball revolution frequency is $118.4 \mathrm{~Hz}$, and the meshing frequency $f_{m}$ is mainly dominant, and the peak of the first frequency is relatively high. At the same time, a sideband appears on both sides of the meshing frequency, and the distance of the sideband from the meshing frequency is the frequency at which the ball revolves.

Compared with healthy gears, sidebands appear around the meshing frequency in the spectrum of root crack failure, as shown in Figure 10. This is because the sun gear shaft frequency $f_{s}$ produces a frequency modulation effect on the meshing frequency. The distance between the sideband and the meshing frequency is $n f_{s}(n=1,2,3, \ldots)$. At the same time, the amplitude of the meshing frequency of the system increases.

\section{Experimental Analysis}

Taking the planetary transmission gearbox as the experimental object, the test was carried out on the planetary gearbox with the sun gear root crack failure. The test bench and measurement points are shown in Figure 11. Measuring points 1-3 are set on the output bearing, ring gear, and input bearing, respectively. The vibration acceleration signals of the healthy and faulty transmission systems are acquired and the vibration signal in the $z$ direction of the measuring point 1 are shown in Figure 12.

The spectrum of the vibration acceleration signals are shown in Figure 13. In the spectrum diagram of the healthy transmission system, the meshing frequency $f_{m}$ and its multiplication are mainly dominant; the meshing frequency of the system is $334.6 \mathrm{~Hz}$, and the frequency of the bearing 


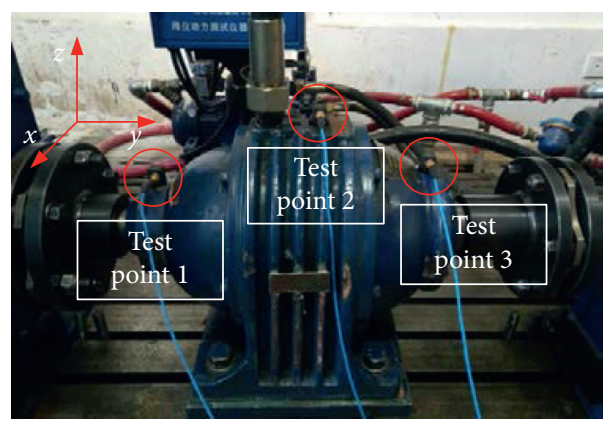

FIgURE 11: Test setup.

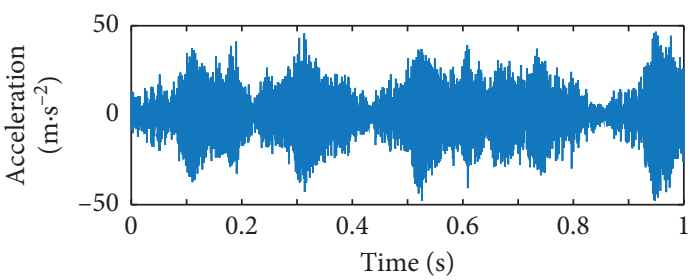

(a)

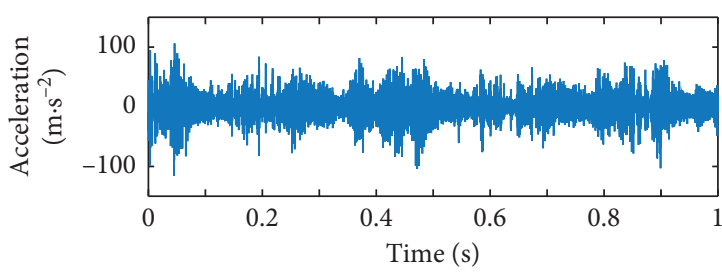

(b)

FiguRE 12: Vibration acceleration of test point 1. (a) Healthy system. (b) Fault system.

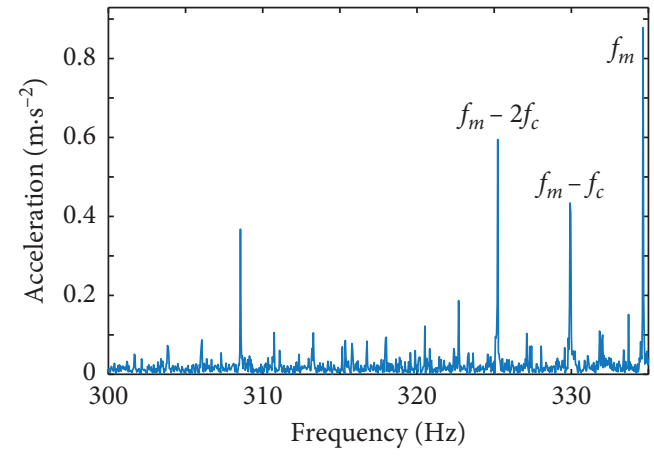

(a)

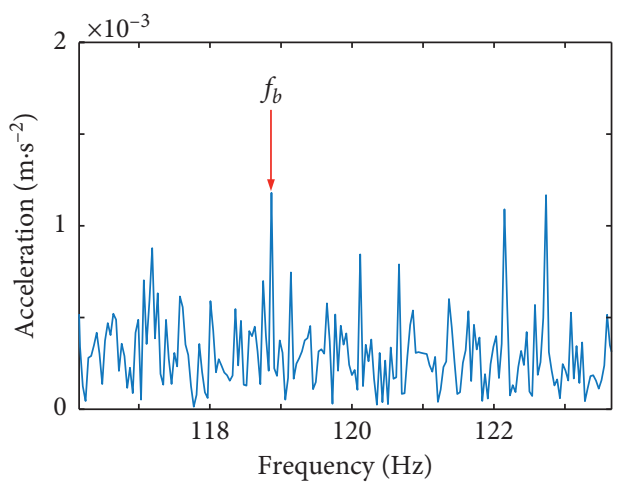

(b)

FIGURE 13: Spectrum diagram of the healthy system. (a) System meshing frequency. (b) Frequency conversion bearing steel ball.

steel ball is $118.9 \mathrm{~Hz}$. At the same time, a sideband having a carrier frequency $f_{\mathrm{c}}$ as a modulation signal appears around the meshing frequency.

Because the influence of the vibration signal transmission path is not considered, there is no sideband caused by the frequency $f_{c}$ modulation of the carrier around the meshing frequency, and the spectrum of the actual vibration signal is slightly different.

As shown in Figure 14, the spectrum of the vibration response of the fault-containing system is obtained. The amplitude of the faulty system spectrum is larger than that of the healthy system. The meshing frequency of the system is $334.6 \mathrm{~Hz}$. The sideband of the sun frequency $f_{\mathrm{s}}$ is modulated around the meshing frequency. The correctness of the simulation model is verified.

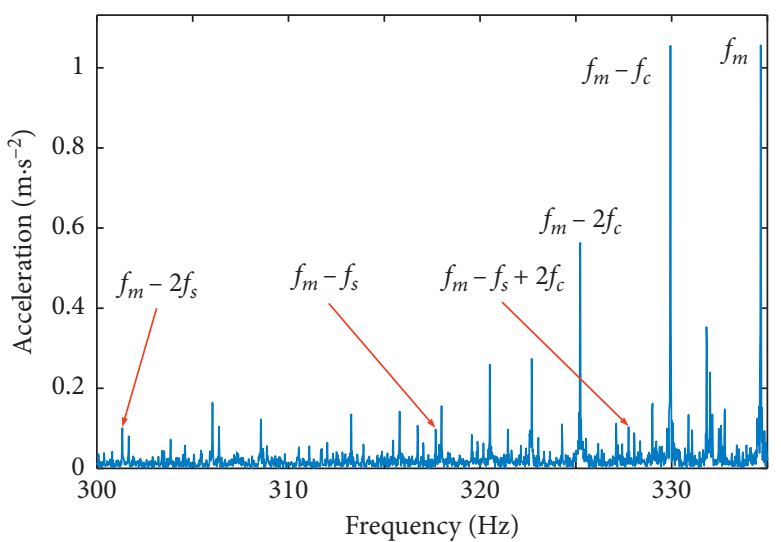

FIGURE 14: Spectrum diagram of the faulty system. 


\section{Conclusions}

The gear-bearing coupling dynamics model of the planetary transmission system is established by using the lumped parameter method. The natural characteristics analysis of the system is carried out, and the influencing factors such as the time-varying parameters of the meshing stiffness and the bearing stiffness are considered. The dynamic characteristics of the planetary transmission system are calculated and simulated. The system dynamic response and frequency domain characteristics are obtained by theoretical analysis and bench test. The next work is to analyze the influence of design and operation parameters on system dynamics, so as to propose effective methods for controlling system vibration. The specific conclusions are as follows:

(1) Based on the analytical method, the natural frequency and modal characteristics of the gear transmission system are obtained. The natural frequencies of the system are mainly concentrated in the 1104 to $23060 \mathrm{~Hz}$.

(2) By solving the dynamic model and comparing and analyzing the vibration response of the transmission system, it is shown that the vibration of the transmission system increases due to the influence of the root crack. In the frequency domain, in the middle of the spectrum of the faulty transmission system, a sideband occurs around the meshing frequency. This is because the frequency shift $f_{s}$ of the sun gear has a modulation effect on the meshing frequency $f_{m}$.

(3) Through the bench experiment, we can find the sidebands generated by the modulation of the carrier frequency $f_{\mathfrak{c}}$, in the spectrogram of the vibration acceleration of the faulty system, and also there are sidebands generated by the frequency $f_{\mathrm{s}}$ modulation of the sun gear. The experimental results verify the correctness of the mathematical model. It also guides the future direction to control nonlinear dynamics of the planetary gear system.

\section{Data Availability}

The data used to support the findings of this study are available from the corresponding author upon request.

\section{Conflicts of Interest}

The authors declare that they have no conflicts of interest.

\section{Acknowledgments}

This work was supported by the National Natural Science Foundation of China (Grant no. 51965025).

\section{References}

[1] Q. Zhu, L. Liu, S. Li, and W. Zhang, "Control of complex nonlinear dynamic rational systems," Complexity, vol. 2018, Article ID 8953035, 12 pages, 2018.
[2] Q. M. Zhu, D. Y. Zhao, and J. Zhang, "A general U-block model-based design procedure for nonlinear polynomial control systems," International Journal of Systems Science, vol. 47, no. 14, pp. 1-10, 2016.

[3] G. Bonori and F. Pellicano, "Non-smooth dynamics of spur gears with manufacturing errors," Journal of Sound and Vibration, vol. 306, no. 1-2, pp. 271-283, 2007.

[4] F. Chaari, T. Fakhfakh, and M. Haddar, "Analytical modelling of spur gear tooth crack and influence on gearmesh stiffness," European Journal of Mechanics-A/Solids, vol. 28, no. 3, pp. 461-468, 2009.

[5] Y. Guo and R. G. Parker, "Dynamic modeling and analysis of a spur planetary gear involving tooth wedging and bearing clearance nonlinearity," European Journal of Mechanics-A/ Solids, vol. 29, no. 6, pp. 1022-1033, 2010.

[6] Z. Chen and Y. Shao, "Dynamic simulation of planetary gear with tooth root crack in ring gear," Engineering Failure Analysis, vol. 31, no. 9, pp. 8-18, 2013.

[7] Q. Zhou, A Dynamic Analysis and Numerical Simulation of Cylindrical Spur Gear and Crack Fault, Ph.D thesis, Taiyuan University of Technology, Taiyuan, China, 2008.

[8] Z. Wan, "Time-varying mesh stiffness algorithm correction and tooth crack dynamic modeling," Journal of Mechanical Engineering, vol. 49, no. 11, p. 153, 2013.

[9] C. T. Walters, "The dynamics of ball bearings," Journal of Tribology, vol. 93, no. 1, pp. 1-10, 1970.

[10] T. A. Harris and M. N. Kotzalas, Advanced Concepts of Bearing Technology: Rolling Bearing Analysis, CRC Press, Boca Raton, FL, USA, 5th edition, 2007.

[11] Z. G. Zhou, Study on Dynamics and Time-dependent Reliability of Gear Transmission System of Wind Turbine under Random Wind Conditions, Ph.D thesis, Chongqing University, Chongqing, China, 2012.

[12] O. D. Mohammed, M. Rantatalo, J. O. Aidanpää, and U. Kumar, "Vibration signal analysis for gear fault diagnosis with various crack progression scenarios," Mechanical Systems \& Signal Processing, vol. 41, no. 1-2, pp. 176-195, 2013.

[13] J. Park, Y. Kim, K. Na, and B. D. Youn, "Variance of energy residual (VER): an efficient method for planetary gear fault detection under variable-speed conditions," Journal of Sound and Vibration, vol. 453, no. 4, pp. 253-267, 2019. 